\title{
Влияние условий осаждения из паров металлоорганических соединений на получение эпитаксиальных слоев $n$-CdTe с использованием изопропилиодида
}

\author{
(C) А.Н. Моисеев, В.С. Евстигнеев, А.В. Чилясов ", М.В. Костюнин \\ Институт химии высокочистых веществ им. Г.Г. Девятых Российской академии наук, \\ 603951 Нижний Новгород, Россия \\ ฯ E-mail: chil@ihps-nnov.ru
}

Поступила в Редакцию 12 ноября 2021 г.

В окончательной редакции 15 ноября 2021 г.

Принята к публикации 15 ноября 2021 г.

Исследовано вхождение иода из изопропилиодида в слои СdTe в процессе металлоорганической парофазной эпитаксии в зависимости от условий осаждения. Рост слоев вели из паров диметилкадмия (ДМК) и диэтилтеллура (ДЭТ) в потоке водорода в вертикальном реакторе с зоной предварительного распада при общем давлении 20 кПа. Общую концентрацию иода определяли методом вторичной ионной масс-спектрометрии, электрически активную концентрацию - из измерения эффекта Холла. Вхождение иода зависит от кристаллографической ориентации подложки (изучены (100), (310), (111)A, (111)B, (211)А и (211)В), концентрации легирующего прекурсора (интервал потоков $5 \cdot 10^{-8}-3 \cdot 10^{-6}$ моль/мин), соотношения металлоорганических соединений (ДМК/ДЭТ $=0.25-4)$, температуры роста $\left(335-390^{\circ} \mathrm{C}\right)$ и стенок реактора выше пьедестала (зона прекрекинга $290-320^{\circ} \mathrm{C}$ ). Общая концентрация иода достигала $5 \cdot 10^{18} \mathrm{~cm}^{-3}$, а степень его активации $\sim 4 \%$. После последующего термического отжига в парах кадмия при $500^{\circ} \mathrm{C}$ степень активации повышалась до $\sim 100 \%$.

Ключевые слова: эпитаксиальные слои, металлоорганическая парофазная эпитаксия, СdТе, легирование иодом, изопропилиодид, термический отжиг.

DOI: 10.21883/FTP.2022.03.52123.9767

\section{1. Введение}

Теллурид кадмия применяется при создании высокочувствительных детекторов $\gamma$ - и рентгеновского излучения, тонкопленочных солнечных элементов и других оптоэлектронных устройств [1,2]. Также CdTe имеет близкий параметр решетки с $\mathrm{Hg}_{1-x} \mathrm{Cd}_{x} \mathrm{Te}$ и поэтому используется в качестве буферного слоя при эпитаксии слоев $\mathrm{Hg}_{1-x} \mathrm{Cd}_{x} \mathrm{Te}$ большой площади на альтернативных подложках, таких как $\mathrm{Si}$ и GaAs [3-6].

Для применения в производстве полупроводниковых устройств необходим материал $n$ - и $p$-типа проводимости с контролируемыми электрофизическими свойствами. Например, для эффективных солнечных элементов требуется $p$-СdТе с концентрацией дырок $\geq 10^{16} \mathrm{~cm}^{-3}$, и в $[7,8]$ мы продемонстрировали возможность получения методом металлоорганической парофазной эпитаксии (MOVPE) легированных мышьяком слоев $\mathrm{CdTe}$ с $p\left(T_{\text {room }}\right)=(1-2) \cdot 10^{17} \mathrm{~cm}^{-3}$. В то же время для детекторов рентгеновского и $\gamma$-излучения на основе структуры $p$-CdTe $/ n-\mathrm{CdTe} / n^{+}-\mathrm{Si}$ требуется высоколегированный $n$-CdTe [9]. В качестве легирующих примесей $n$-типа в эпитаксии CdTe наиболее широко используются иод и индий. Однако использование иода предпочтительнее, поскольку он обладает меньшим значением коэффициента диффузии в CdTe. Также отмечено, что в MOVPE алкилы индия реагируют с алкилами Те и образуют аддукты с низкой летучестью, которые приводят к эффектам памяти аппаратуры. В то же время использование алкилов иода не приводит к эффектам памяти [6]. При осаждении CdTe из газовой фазы в качестве источников иода применяется этилиодид [6,9-11], а также изопропилиодид и н-бутилиодид при осаждении $\mathrm{Hg}_{1-x} \mathrm{Cd}_{x} \mathrm{Te}[12,13]$. В сравнении с этилиодидом, два последних прекурсора менее термически стойкие, а также имеют более низкое давление насыщенного пара, что упрощает дозирование примеси при низком уровне легирования слоев.

Иод в CdTe является мелким донором с энергией ионизации 10-15 мэВ, определенной из температурной зависимости холловских измерений и фотолюминесценции $[9,14,15]$. Для того чтобы иод проявлял донорные свойства, ему необходимо занимать места в подрешетке теллура $\left(\mathrm{I}_{\mathrm{Te}}\right)$. Следовательно, необходимо проводить синтез слоев CdTe в избытке кадмия, что приведет к увеличению концентрации вакансий теллура $\left(V_{\mathrm{Te}}\right)$ и, соответственно, к увеличению концентрации иода в слоях. Помимо этого, рост CdTe в избытке кадмия способствует уменьшению концентрации вакансий кадмия $\left(V_{\mathrm{Cd}}\right)$, которые могут компенсировать иод путем образования комплексов $V_{\mathrm{Cd}}-\mathrm{I}_{\mathrm{Te}}$.

Исследования по легированию СdТе этилиодидом в MOVPE было проведено в ряде работ [9-11]. В [9] рост $\mathrm{CdTe}$ проводили из диметилкадмия (ДМК), диэтилтеллура (ДЭТ) и диизопропилтеллура (ДИПТ) в вертикальном реакторе. Осаждение проводили на подложки GaAs (100) в диапазоне температур 300-450 $\mathrm{C}$, при нагретой стенке реактора выше пьедестала $200-300^{\circ} \mathrm{C}$ и 
атмосферном давлении. Мольные отношения ДЭТ/ДМК и ДИПТ/ДМК были фиксированы и составляли 0.5. Концентрация электронов в слоях увеличивалась с понижением температуры роста с 425 до $325^{\circ} \mathrm{C}$. Легированные слои, осажденные из ДЭТ, имели более высокую концентрацию электронов, чем слои, выращенные с использованием ДИПТ. Максимальная концентрация электронов составила $2.6 \cdot 10^{18} \mathrm{~cm}^{-3}$ при температуре подложки и стенки реактора 325 и $250^{\circ} \mathrm{C}$ соответственно. В [10] осаждение проводили из паров ДМК, ДЭТ и этилиодида на подложках $n^{+}-\mathrm{Si}(211)$ в диапазоне температур $325-450^{\circ} \mathrm{C}$ и атмосферном давлении. Мольное отношение ДЭТ/ДМК изменяли от 0.05 до 0.3. Концентрация электронов постепенно увеличивалась при уменьшении соотношения ДЭТ/ДМК. Наибольшая концентрация электронов была $2.5 \cdot 10^{18} \mathrm{~cm}^{-3}$ в слоях, полученных при соотношении ДЭТ/ДМК $=0.05$ и температуре подложки $400^{\circ} \mathrm{C}$.

В [11] при создании $n-p$-гомоперехода для солнечных элементов $n$-CdTe/ $p$-CdTe/ZnTe/GaAs (100) авторы использовали метод MOVPE с легированием CdTe мышьяком из арсина и иодом из этилиодида в вертикальном реакторе с холодными стенками. Осаждение $n$-CdTe вели при давлении 100 и 500 Торр из паров ДМК и ДИПТ (ДМК/ДИПТ $=1-7$ ) при температурах 250, 300 и $400^{\circ} \mathrm{C}$. Свойства слоев $\mathrm{CdTe}$ сильно зависели от условий осаждения. Наибольшая концентрация свободных электронов $4.5 \cdot 10^{16} \mathrm{~cm}^{-3}$ (без дополнительного отжига при $550^{\circ} \mathrm{C}$ в атмосфере кадмия) была получена при осаждении слоев при температуре $250^{\circ} \mathrm{C}$, давлении в реакторе 500 Торр и соотношении ДМК/ДИПТ $=7$. Сравнение общего содержания иода (метод вторичной ионной масс-спектрометрии ВИМС) и концентрации свободных электронов (измерение эффекта Холла) в слоях показало, что степень активации иода составляла $25-30 \%$ при температуре осаждения $250-300^{\circ} \mathrm{C}$.

Из анализа литературных данных следует, что вхождение примеси иода в CdTe и его электрическое поведение сильно зависят от условий получения, а использование для легирования в MOVPE CdTe менее термически стойких, чем этилиодид, соединений не изучалось.

В данной работе мы исследовали закономерности вхождения иода из изопропилиодида в MOVPE CdTe и его электрической активности в зависимости от кристаллографической ориентации подложки, концентрации легирующего прекурсора, соотношения металлоорганических соединений, температуры роста и стенок реактора, а также влияние последующего термического отжига на концентрацию носителей заряда в слоях.

\section{2. Методика эксперимента}

Слои CdTe были выращены в вертикальном кварцевом реакторе с зоной предварительного нагрева выше пьедестала $\left(T_{\text {prezon }}=290-320^{\circ} \mathrm{C}\right)$ при общем давлении 20 кПа и температуре подложки $T_{\text {subs }}=335-390^{\circ} \mathrm{C}$ (внутренний резистивный нагреватель). В качестве источников кадмия и теллура использовали ДМК и ДЭТ соответственно. Легирование слоев иодом осуществляли с использованием изопропилиодида (ИПИ). Чистота ДМК, ДЭТ составляла не менее $99.999 \%$, ИПИ не менее 98\%. При осаждении слоев СdТе потоки ДЭТ $\left(V_{\mathrm{DETe}}\right)$ и ДМК $\left(V_{\mathrm{DMCd}}\right)$ изменяли от $4 \cdot 10^{-5}$ до $1.6 \cdot 10^{-4}$ моль/мин, а соотношение ДМК/ДЭТ на входе в реактор от 0.25 до 4. Величину потока ИПИ $\left(V_{\mathrm{IPI}}\right)$ варьировали в диапазоне от $5 \cdot 10^{-8}$ до $3 \cdot 10^{-6}$ моль/мин. Ввод реагентов в реактор осуществляли по раздельным трубкам в потоке водорода, очищенного диффузией через палладиевый фильтр. Общий поток водорода в реактор составлял 3 л/мин (в нормальных условиях). Для роста использовали „еріready“ подложки полуизолирующего GaAs c кристаллографическими ориентациями $(100) 4^{\circ} \rightarrow\langle 110\rangle,(310),(111)$ (А и В) и (211)(А и В). Перед осаждением слоев подложки отжигали в потоке водорода при $550^{\circ} \mathrm{C}$ в течение 15 мин для удаления слоя естественного окисла. Для предотвращения диффузии компонентов подложки в легированный слой первоначально на подложки наносили нелегированный буферный слой $\mathrm{CdTe}$ толщиной $2-3$ мкм.

Толщину слоев CdTe с погрешностью 5\% определяли из интерференционных кривых, полученных на фурьеспектрометре Nicolet 6700. Инфракрасные спектры слоев записывались при $298 \mathrm{~K}$ в диапазоне $500-6000 \mathrm{~cm}^{-1}$ с разрешением $0.5 \mathrm{~cm}^{-1}$. Профиль концентрации иода по толщине слоев СdTе исследовали методом ВИМС. Для регистрации спектров ВИМС использовали времяпролетный масс-спектрометр вторичных ионов TOF.SIMS-5. Концентрационные профили были получены с использованием импульсного ионного пучка $\mathrm{Bi}_{3}^{+}$с энергией 25 кэВ (ток 1 пА, растр $30 \times 30$ мкм) для анализа и $\mathrm{Cs}^{+}$ с энергией 1 кэВ (растр $150 \times 150$ мкм) для распыления. Регистрировались линии ионов ${ }^{127} \mathrm{I}$ и шумовой сигнал от линий ${ }^{126} \mathrm{Te}$ и ${ }^{128} \mathrm{Te}$. Нормированное содержание иода в слоях определялось отношением разницы интенсивностей этих линий к интенсивности кластерной линии $\mathrm{CdTe}$. Количественное содержание иода определяли с помощью атомно-эмиссионной спектроскопии с индуктивно связанной плазмой (относительная погрешность 5\%).

Темновые электрофизические параметры слоев CdTe с погрешностью $20 \%$ определяли из измерений эффекта Холла методом Ван-дер-Пау в постоянном магнитном поле 0.5 Тл при комнатной температуре. Толщина легированных слоев для электрофизических измерений составляла не менее 3 мкм. Для создания омических контактов к $n$-СdТе использовали индий.

Кристаллографическую ориентацию и структурное совершенство слоев определяли по полуширине кривой качания (FWHM) методом двухкристальной рентгеновской дифракции на приборе ДРОН-4М.

Для увеличения доли (концентрации) электрически активного иода проводили высокотемпературные отжиги CdTe в парах кадмия в диапазоне температур $400-600^{\circ} \mathrm{C}$ в течение 3 ч. Навеску $\mathrm{Cd}$ и образцы $\mathrm{CdTe}$ 
Таблица 1. Некоторые свойства гетероэпитаксиальных слоев $\mathrm{CdTe}: \mathrm{I} / \mathrm{CdTe} / \mathrm{GaAs}$ различной кристаллографической ориентации, осажденных в одном процессе $\left(T_{\text {subs }}=350^{\circ} \mathrm{C}, T_{\text {prezon }}=300^{\circ} \mathrm{C}, V_{\mathrm{DMCd}}=V_{\mathrm{DETe}}=4 \cdot 10^{-5}\right.$ моль $/$ мин, $V_{\mathrm{IPI}}=1 \cdot 10^{-6} \mathrm{моль} /$ мин $)$

\begin{tabular}{l|c|c|c|c|c|c}
\hline \multirow{2}{*}{ Свойство } & \multicolumn{5}{|c}{ Ориентация } \\
\cline { 2 - 7 } & $(100)$ & $(310)$ & $(111) \mathrm{B}$ & $(111) \mathrm{A}$ & $(211) \mathrm{B}$ & $(211) \mathrm{A}$ \\
\hline Поверхность & Зеркальная & Зеркальная & Зеркальная & Очень матовая & Зеркально-матовая & Матовая \\
FWHМ, угл. мин & 7.4 & 6.1 & $11.1^{*}$ & $6.4^{* *}$ & 3.6 & 1.9 \\
$n_{\text {room }}, \mathrm{cm}^{-3}$ & $5.4 \cdot 10^{15}$ & $6.6 \cdot 10^{14}$ & $2.3 \cdot 10^{12}$ & $8.5 \cdot 10^{12}$ & $1 \cdot 10^{12}$ & $1.1 \cdot 10^{16}$ \\
$\mu_{\text {room }}, \mathrm{cm}^{2} /(\mathrm{B} \cdot \mathrm{c})$ & 240 & 70 & 110 & 90 & 370 & 330
\end{tabular}

Примечание. ${ }^{*}$ Имеются двойники $(\sim 50 \%)$. ${ }^{* *}$ Имеются двойники $(\sim 20 \%)$.

помещали в кварцевую ампулу и вакуумировали, а затем загружали в двухзонную печь. Температура зоны с навеской $\mathrm{Cd}$ была на $10^{\circ} \mathrm{C}$ ниже температуры зоны с образцами. Для корректной интерпретации свойств легированных иодом слоев $\mathrm{CdTe}$ использовали сравнение с нелегированными образцами, выращенными и отожженными в аналогичных условиях.

\section{3. Экспериментальные результаты и их обсуждение}

В настоящей работе для изучения вхождения примеси иода в эпитаксиальные слои CdTe был существенно расширен круг исследуемых ориентаций, но основное внимание, как и в работах других авторов, уделено слоям $\mathrm{CdTe}(100)$. В табл. 1 приведены некоторые характеристики полученных в одном процессе слоев $\mathrm{CdTe}$, ориентация которых всегда совпадала с ориентацией подложки. Все указанные в таблице свойства слоев заметно зависят от ориентации. Морфология поверхности образцов менялась от зеркальной для ориентаций (100) и (310) до матовой для (111)А и (211)A, наилучшая полуширина кривой качания наблюдалась у образца CdTe (211)A. Концентрация свободных (некомпенсированных) электронов максимальна в слоях $\mathrm{CdTe}(211) \mathrm{A}$ $\left(1.1 \cdot 10^{16} \mathrm{~cm}^{-3}\right)$.

Вхождение иода в CdTe также сильно зависит от кристаллографической ориентации растущего слоя (рис. 1). Ориентационный эффект вхождения примесей уже наблюдался в более ранних работах при легировании слоев соединений $\mathrm{A}^{\mathrm{III}} \mathrm{B}^{\mathrm{V}}$ из газовой фазы $[16,17]$, а также при легировании мышьяком слоев $\mathrm{Hg}_{1-x} \mathrm{Cd}_{x} \mathrm{Te}$ и $\mathrm{CdTe}[3,18]$. Наибольшее вхождение иода $\sim 5 \cdot 10^{18} \mathrm{~cm}^{-3}$ наблюдается для ориентации (111)А (рис. 1), которое практически на 2 порядка выше, чем для ориентации (100). Концентрация иода в слоях CdTe разной кристаллографической ориентации увеличивается в ряду $(100)<(310)<(111)$ В < (111)А. При этом скорость роста слоев $\mathrm{CdTe}$ на различных плоскостях убывает в ряду $(111)$ В $>(100)>(310) \sim(111)$ А. Различие скорости роста на поверхности разной ориентации обусловлено кинетикой поверхностных процессов и определяется различной геометрией связей, образующихся между адсорбированными атомами и поверхностью с

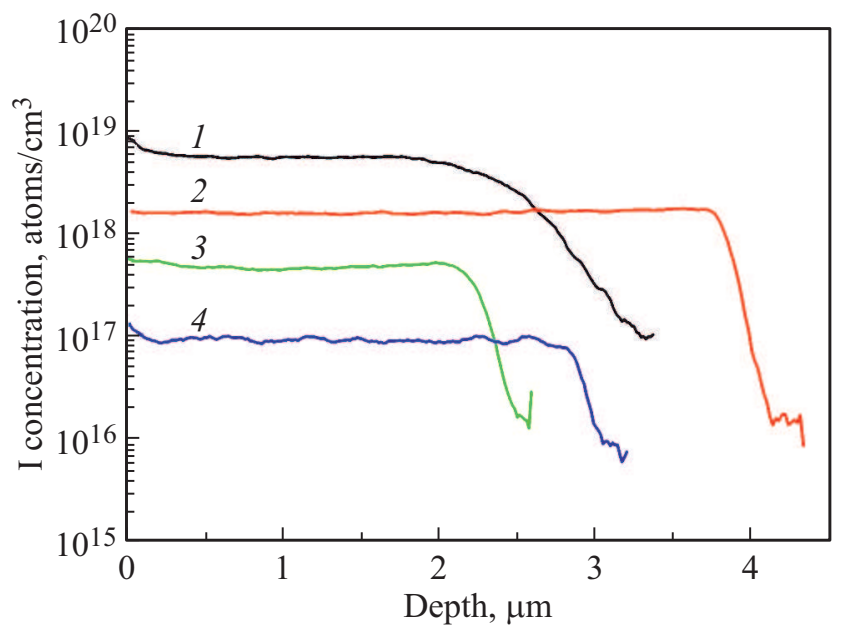

Рис. 1. Концентрационное распределение иода по толщине слоев СdТе разной кристаллографической ориентации, выращенных в одном процессе: $1-(111) \mathrm{A}, 2-(111) \mathrm{B}, 3-(310)$, $4-(100) . \quad\left(T_{\text {subs }}=350^{\circ} \mathrm{C}, \quad T_{\text {prezon }}=300^{\circ} \mathrm{C}, \quad\right.$ ДМК $/$ ДЭТ $=1$, $V_{\mathrm{DMCd}}=4 \cdot 10^{-5}$ моль/мин, $V_{\mathrm{IPI}}=5 \cdot 10^{-7}$ моль $/$ мин $)$.

более благоприятной кинетикой роста на ориентации (111)В. Однако в наших условиях вхождение иода в CdTe не коррелирует со скоростью роста. Процесс вхождения иода в CdTe следует рассматривать как процесс, конкурирующий с вхождением Те. Поскольку поверхность (111)В является Те-обогащенной, а (111)А Cd-обогащенной, то на поверхности ориентации (111)A больше вакантных теллуровых мест, которые может занимать иод. Помимо этого, существенный вклад во вхождение примеси в слой может вносить процесс распада легирующего соединения [19], а также процесс десорбции иода с поверхности. Для каждой ориентации $\mathrm{CdTe}$ вклад этих стадий в процесс вхождения иода в слой может быть различен и зависит от других условий роста, прежде всего температуры подложки и состава подлетающей к ней парогазовой смеси.

На рис. 2 представлена зависимость вхождения иода в $\mathrm{CdTe}(100)$, полученного при фиксированном потоке ИПИ $5 \cdot 10^{-7}$ моль/мин и изменении в едином процессе осаждения мольного отношения ДМК/ДЭТ (при фиксированном значении потока ДМК). Как видно из рис. 2, с увеличением ДМК/ДЭТ концентрация иода в 


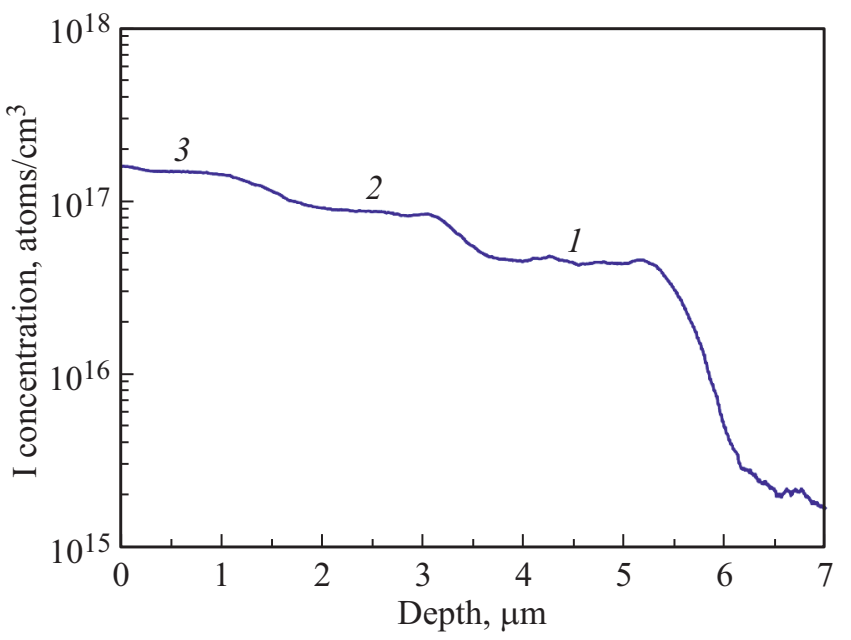

Рис. 2. Концентрационное распределение иода по толщине $\mathrm{CdTe}(100)$, полученного при изменении в процессе осаждения мольного отношения ДМК/ДЭТ: $1-0.5,2-1,3-$ 1.4. $\left(T_{\text {subs }}=350^{\circ} \mathrm{C}, T_{\text {prezon }}=300^{\circ} \mathrm{C}, V_{\mathrm{DMCd}}=4 \cdot 10^{-5}\right.$ моль $/$ мин, $V_{\mathrm{IPI}}=5 \cdot 10^{-7}$ моль/мин).

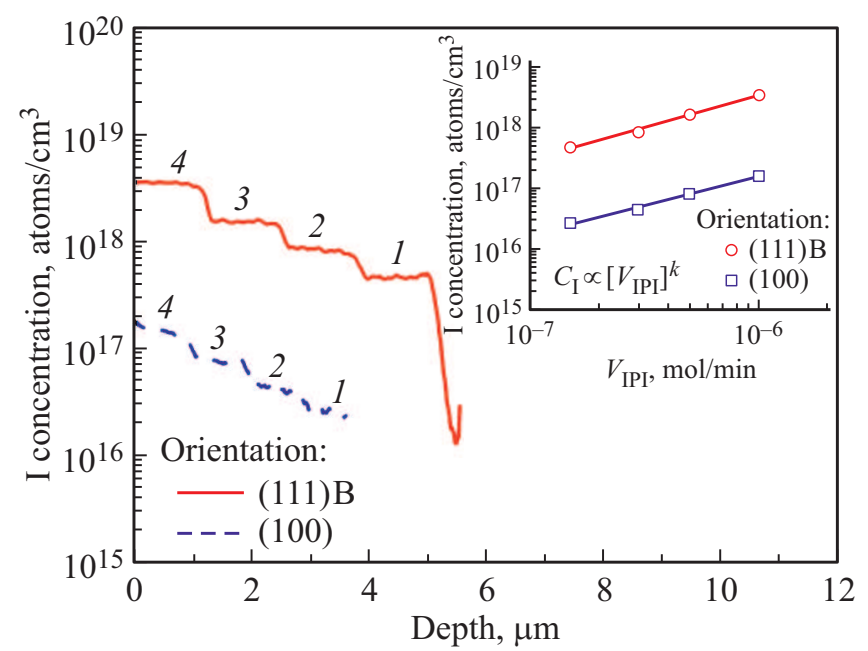

Рис. 3. Концентрационное распределение иода по толщине слоев CdTe разной кристаллографической ориентации, выращенных в одном процессе при ступенчатом измене-

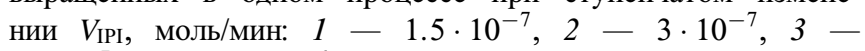
$5 \cdot 10^{-7}, 4-1 \cdot 10^{-6}$; вхождение иода в СdTе как функция потока ИПИ для ориентаций $(111) \mathrm{B}$ и $(100) 4^{\circ} \rightarrow\langle 110\rangle$ (см. вставку). $\left(T_{\text {subs }}=350^{\circ} \mathrm{C}, T_{\text {prezon }}=300^{\circ} \mathrm{C}\right.$, ДМК $/ Д Э Т ~=1$, $V_{\mathrm{DMCd}}=4 \cdot 10^{-5}$ моль/мин).

слоях монотонно увеличивается. Увеличение отношения ДМК/ДЭТ в процессе роста приводит к увеличению концентрации вакансий теллура и увеличению вероятности встраивания иода в подрешетку теллура.

На рис. 3 представлено концентрационное распределение иода в слоях $\mathrm{CdTe}$, полученных в одном процессе на подложках ориентаций (100) и (111)В при ступенчатом изменении потока ИПИ от $1.5 \cdot 10^{-7}$ до $1 \cdot 10^{-6}$ моль/мин и одинаковом мольном отношении
ДМК/ДЭТ $=1$. Повышение потока ИПИ приводит к увеличению концентрации иода в слоях. Вхождение иода в слои СdTе кристаллографических ориентаций (100) и (111)В в диапазоне потоков ИПИ в газовой фазе $1.5 \cdot 10^{-7}-1 \cdot 10^{-6}$ моль/мин увеличивается согласно выражению (см. вставку на рис. 3 ):

$$
C_{\text {I }}\left(\text { ат } / \mathrm{cm}^{3}\right) \propto\left[V_{\text {IPI }}(\text { моль } / \text { мин })\right]^{k},
$$

где $k=0.95-1.05( \pm 0.05)$.

На рис. 4 представлена зависимость вхождения иода в CdTe (310), полученного при фиксированном потоке ИПИ $1 \cdot 10^{-6}$ моль/мин и изменении в процессе осаждения температуры роста от 350 до $390^{\circ} \mathrm{C}$. Как видно из рис. 4 , хорошо различимы четыре ступеньки легирования. Наибольшее вхождение иода наблюдается при температуре роста $350^{\circ} \mathrm{C}$. Постепенное повышение температуры роста до $390^{\circ} \mathrm{C}$ приводит к уменьшению концентрации иода в слое. Мы полагаем, что на уменьшение вхождения иода в CdTe при повышении температуры роста оказывают влияние два фактора: 1) увеличение степени термического разложения ДЭТ и увеличение соотношения $\mathrm{Te} / \mathrm{Cd}$ на поверхности; 2) увеличение десорбции иода с ростовой поверхности. Подобный эффект был отмечен нами при легировании СdTе мышьяком [8], а также в ранних работах по легированию соединений $\mathrm{A}^{\mathrm{III}} \mathrm{B}^{\mathrm{V}}$ из газовой фазы [20].

Была исследована зависимость концентрации атомов иода и электрофизических параметров в слоях CdTe:I (100) от потока изопропилиодида (табл. 2). Концентрация свободных электронов в слоях проходит через максимум $5.6 \cdot 10^{15} \mathrm{~cm}^{-3}$ при потоке изопропилиодида $1 \cdot 10^{-6}$ моль/мин. Качественно наш результат повторяет данные [9] о наличии максимума на аналогичной зависимости, однако количественно заметно отличается. Отличие может быть обусловлено различием прекурсоров,

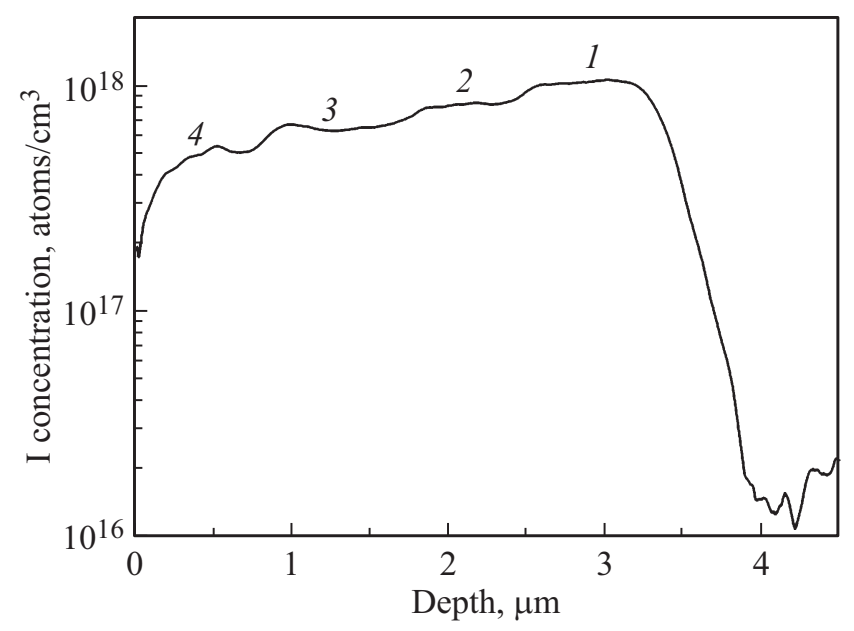

Рис. 4. Концентрационное распределение иода по толщине $\mathrm{CdTe}(310)$, полученного при изменении в процессе осаждения температуры роста, ${ }^{\circ} \mathrm{C}: 1-350,2-365,3-380$, 4 - 390. $\left(T_{\text {prezon }}=300^{\circ} \mathrm{C}\right.$, ДМК/ДЭТ $=1, \quad V_{\mathrm{DMCd}}=4 \cdot 10^{-5}$ моль/мин, $V_{\mathrm{IPI}}=1 \cdot 10^{-6}$ моль/мин). 
Таблица 2. Зависимость концентрации иода $C(\mathrm{I})_{\mathrm{CdTe}}$ (ВИМС) и электрофизических параметров ( $n$ и $\mu)$ слоев CdTe: I (100) от величины потока легирующего соединения $V_{\mathrm{IPI}}\left(T_{\text {subs }}=350^{\circ} \mathrm{C}\right.$, $T_{\text {prezon }}=300^{\circ} \mathrm{C}, V_{\mathrm{DMCd}}=V_{\mathrm{DETe}}=4 \cdot 10^{-5}$ моль $/$ мин $)$

\begin{tabular}{|c|c|c|c|c|}
\hline$V_{\text {IPI, }}$ моль/мИн & $5 \cdot 10^{-8}$ & $5 \cdot 10^{-7}$ & $1 \cdot 10^{-6}$ & $3 \cdot 10^{-6}$ \\
\hline$C(\mathrm{I})_{\mathrm{CdTe}}, \mathrm{aT} / \mathrm{cm}^{3}$ & $8.3 \cdot 10^{15}$ & $7.7 \cdot 10^{16}$ & $1.5 \cdot 10^{17}$ & $4.3 \cdot 10^{17}$ \\
\hline$n_{\text {room }}, \mathrm{cm}^{-3}$ & $1.4 \cdot 10^{12}$ & $7.4 \cdot 10^{14}$ & $5.6 \cdot 10^{15}$ & $2.2 \cdot 10^{15}$ \\
\hline$\mu_{\text {room }}, \mathrm{cm}^{2} /(\mathrm{B} \cdot \mathrm{c})$ & 420 & 480 & 240 & 310 \\
\hline $\begin{array}{l}\text { Степень } \\
\text { активации I, \% }\end{array}$ & 0.017 & 0.96 & 3.7 & 0.51 \\
\hline
\end{tabular}

Таблица 3. Зависимость концентрации и подвижности свободных электронов в слоях CdTe:I(100) от соотношения ДМК/ДЭТ в газовой фазе $\left(T_{\text {subs }}=350^{\circ} \mathrm{C}, T_{\text {prezon }}=300^{\circ} \mathrm{C}\right.$, $V_{\mathrm{DETe}}=4 \cdot 10^{-5}$ моль $/$ мин, $V_{\mathrm{IPI}}=5 \cdot 10^{-7}$ моль $/$ Мин $)$

\begin{tabular}{l|c|c|c|c}
\hline \multicolumn{1}{c|}{ ДМК/ДЭТ } & $0.25^{*}$ & 1 & 2 & 3 \\
\hline$n_{\text {room }}, \mathrm{cm}^{-3}$ & $\sim 1 \cdot 10^{12 * *}$ & $7.4 \cdot 10^{14}$ & $3 \cdot 10^{15}$ & $1.8 \cdot 10^{15}$ \\
$\mu_{\text {room }, \mathrm{cm}^{2} /(\mathrm{B} \cdot \mathrm{c})}$ & - & 480 & 540 & 200 \\
Скорость & 2.79 & 1.46 & 1.86 & 1.24 \\
роста, мКм/ч & & & &
\end{tabular}

Примечание. ${ }^{*} V_{\mathrm{DMCd}}=4 \cdot 10^{-5}$ моль $/$ мин, $V_{\mathrm{DETe}}=1.6 \cdot 10^{-4}$ моль $/$ мин. ** Оценка из величины удельного сопротивления $\left(\rho \sim 10^{4} \mathrm{OM} \cdot \mathrm{cm}\right)$.

условий осаждения и геометрии реактора. Сравнение результатов измерения эффекта Холла с концентрацией иода в слоях (ВИМС) показывает, что степень электрической активности иода изменяется в указанных условиях от $\sim 0.02$ до $\sim 4 \%$ с максимумом также при $V_{\text {IPI }}=1 \cdot 10^{-6}$ моль/мин.

В табл. 3 приведены результаты исследования влияния соотношения ДМК/ДЭТ в газовой фазе на концентрацию и подвижность электронов в слоях CdTe:I (100).

В избытке теллура слои были высокоомные, при соотношении ДМК/ДЭТ = 1 и выше концентрация электронов проходила через максимум $3 \cdot 10^{15} \mathrm{~cm}^{-3}$ при ДМК/ДЭТ = 2. Аналогично вела себя и подвижность электронов. Скорость роста слоев в избытке теллура в газовой фазе была заметно выше, чем в избытке кадмия. Увеличение потока легирующего соединения в 2 раза повышало концентрацию электронов в максимуме до $9.8 \cdot 10^{15} \mathrm{~cm}^{-3}$ (рис. 5), а для образца ориентации (211)А достигнуто значение $n=3.6 \cdot 10^{16} \mathrm{~cm}^{-3}$. В работах $[10,11]$, где исследовалась зависимость концентрации свободных электронов в $\mathrm{CdTe}: \mathrm{I}(100)$ от соотношения ДМК/ДЭТ (ДИПТ) в газовой фазе, наличие максимума на этой зависимости не отмечалось.

В работе [9] отмечался довольно резкий максимум на зависимости концентрации свободных электронов в слоях CdTe:I(100) от температуры нагретых стенок реактора при $T_{\text {prezon }}=250^{\circ} \mathrm{C}$. В нашей работе также наблюдался на этой зависимости максимум (рис. 6), но только при $T_{\text {prezon }}=300-310^{\circ} \mathrm{C}$. Понижение температуры подложки ниже $350^{\circ} \mathrm{C}$ в наших условиях не приводи- ло к увеличению концентрации свободных электронов в слоях CdTe:I(100) (табл. 4), как в работах [9-11]. Проведение отжига легированных слоев в атмосфере насыщенных паров кадмия при $500^{\circ} \mathrm{C}$ в течение 3 ч приводило к практически полной активации примеси иода, как показано на примере CdTe:I (100) (табл. 5).

Электрическое поведение иода в слоях CdTe:I мы объясняем, как и авторы работ [9-11,21,22], на основе компенсационной модели. Иод занимает преимущественно места теллура в решетке $\mathrm{CdTe}\left(I_{\mathrm{Te}}\right)$ и образует с вакансиями кадмия заряженный акцепторный комплекс $\left[V_{\mathrm{Cd}}^{2-}-\mathrm{I}_{\mathrm{Te}}^{+}\right]^{-}$. Компенсация электрически активного иода происходит по реакции $\left[V_{\mathrm{Cd}}^{2-}-\mathrm{I}_{\mathrm{Te}}^{+}\right]^{-}+\mathrm{I}_{\mathrm{Te}}^{+}$. Чем выше концентрация вакансий кадмия, тем выше будет кон-

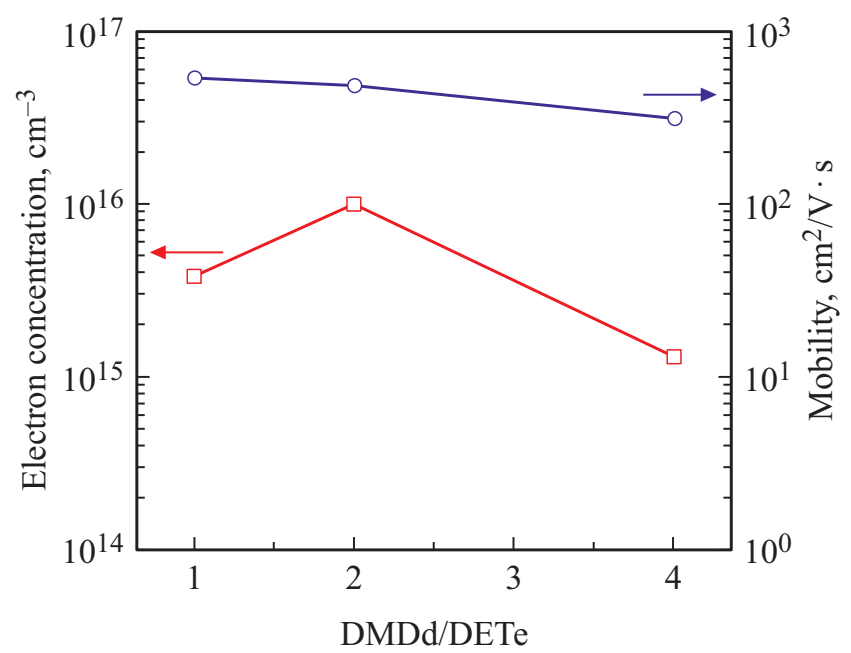

Рис. 5. Зависимость концентрации и подвижности свободных электронов в слоях CdTe:I (100) от соотношения ДМК/ДЭТ в газовой фазе $\left(T_{\text {subs }}=350^{\circ} \mathrm{C}, T_{\text {prezon }}=310^{\circ} \mathrm{C}\right.$, $V_{\text {DETe }}=4 \cdot 10^{-5}$ моль/мин, $V_{\text {IPI }}=1 \cdot 10^{-6}$ моль/мин).

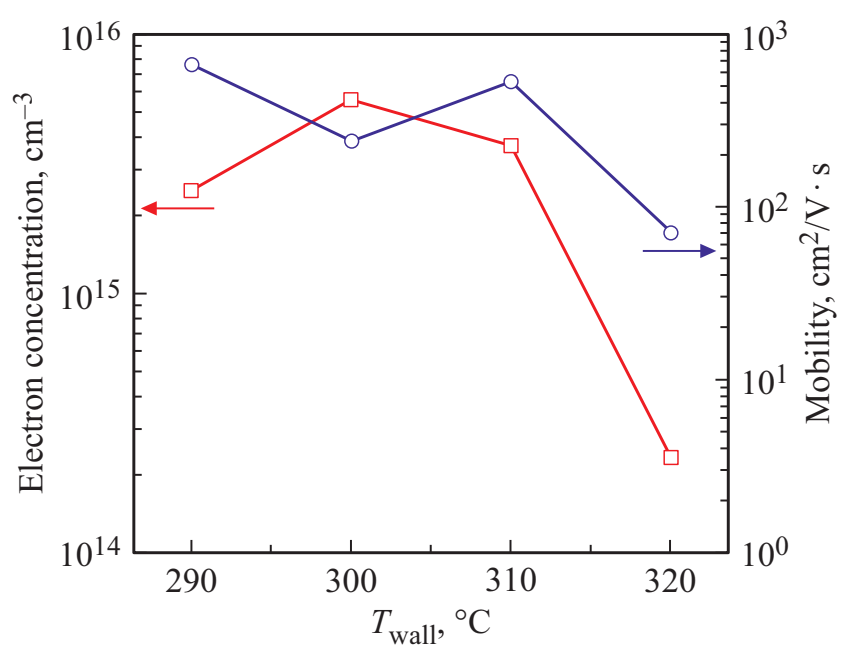

Рис. 6. Зависимость концентрации и подвижности свободных электронов в слоях CdTe:I(100) от температуры стенок реактора $\quad\left(T_{\text {subs }}=350^{\circ} \mathrm{C}, \quad V_{\mathrm{DMCd}}=V_{\mathrm{DETe}}=4 \cdot 10^{-5}\right.$ моль $/$ мин, $V_{\mathrm{IPI}}=1 \cdot 10^{-6}$ моль $/$ мин $)$. 
Таблица 4. Зависимость концентрации и подвижности электронов в слоях CdTe:I(100) от температуры роста $\left(T_{\text {prezon }}=310^{\circ} \mathrm{C}, \quad V_{\mathrm{DMCd}}=8 \cdot 10^{-5}\right.$ моль $/ \mathrm{мин}, \quad V_{\mathrm{DETe}}=$ $=4 \cdot 10^{-5}$ моль/мин, $V_{\mathrm{IPI}}=1 \cdot 10^{-6}$ моль/мин)

\begin{tabular}{l|c|c|c}
\hline$T_{\text {grow }},{ }^{\circ} \mathrm{C}$ & 335 & 350 & 365 \\
$n_{\text {room }}, \mathrm{cm}^{-3}$ & $1.2 \cdot 10^{15}$ & $9.8 \cdot 10^{15}$ & $2.7 \cdot 10^{13}$ \\
$\mu_{\text {room }}, \mathrm{cm}^{2} /(\mathrm{B} \cdot \mathrm{c})$ & 90 & 480 & 60 \\
Скорость роста, мКм/ч & 1.58 & 1.9 & 1.95
\end{tabular}

Таблица 5. Электрофизические параметры слоев $\mathrm{CdTe}$ до и после отжига в парах кадмия в течение 3 ч

\begin{tabular}{|c|c|c|c|}
\hline \multirow[b]{2}{*}{$\begin{array}{l}\text { Условия } \\
\text { отжига }\end{array}$} & \multicolumn{2}{|c|}{ Концентрация носителей заряда $n, \mathrm{~cm}^{-3}$} & \multirow{2}{*}{$\begin{array}{c}\text { Степень } \\
\text { активации } \\
\text { I, \% }\end{array}$} \\
\hline & $\begin{array}{c}\text { CdTe }(100) \\
\text { нелегированный }\end{array}$ & $\begin{array}{c}\text { CdTe }(100): \mathrm{I} \\
C(\mathrm{I})=910^{16} \mathrm{~cm}^{-3}\end{array}$ & \\
\hline Исходные & Высокоомный & $8.0 \cdot 10^{14}$ & 0.9 \\
\hline $400^{\circ} \mathrm{C}$ & Высокоомный & $5.2 \cdot 10^{16}$ & 58 \\
\hline $500^{\circ} \mathrm{C}$ & $3.6 \cdot 10^{15}$ & $8.9 \cdot 10^{16}$ & $95^{*}$ \\
\hline $600^{\circ} \mathrm{C}$ & $1.7 \cdot 10^{16}$ & $8.3 \cdot 10^{16}$ & $73^{*}$ \\
\hline
\end{tabular}

Примечание. ${ }^{*}$ С учетом влияния собственных дефектов после отжига.

центрация комплексов и, следовательно, больше эффект компенсации.

Соотношение $\mathrm{Cd} / \mathrm{Te}$ в газовой фазе и на поверхности растущего слоя может не совпадать и зависит от термической устойчивости прекурсоров и условий осаждения. Диметилкадмий практически полностью распадается в потоке водорода при $350^{\circ} \mathrm{C}$, а начинает распадаться при $\sim 250^{\circ} \mathrm{C}[23]$. Диэтилтеллур начинает распадаться в эквимолярной смеси с диметилкадмием при $330-350^{\circ} \mathrm{C}$, а практически полностью $->400^{\circ} \mathrm{C}$ [24]. Сильное влияние на степень распада ДМК (заметно слабее для ДЭТ) оказывает гетерогенный фактор (соотношение поверхности к объему). Наличие зоны предраспада $\left(T_{\text {prezon }}\right)$ перед пьедесталом увеличивает степень распада металлоорганических соединений (МОС), меняя соотношение $\mathrm{Cd} / \mathrm{Te}$ в подлетающем к подложке потоке. Количественно изменение состава газовой фазы зависит от температуры $T_{\text {prezon }}$ и времени воздействия ее на молекулы реагента при движении смеси от зоны предраспада до пьедестала, а это в свою очередь зависит от линейной скорости потока и геометрии реактора. Таких данных об использованном реакторе большинство авторов не приводят. Различия в условиях роста и в геометрии реактора могут приводить к различию во вхождении и поведении иода в CdTe.

Для слоев CdTe разной кристаллографической ориентации характерно различие в концентрации собственных дефектов, и примесь иода входит в них и ведет себя неодинаково, что подтверждается полученными результатами (табл. 1 и рис. 1). Увеличение потока легирующего соединения при прочих равных условиях приводит к возрастанию количества, введенного в слой иода (см. вставку на рис. 3 и табл. 2). Возрастает при этом и концентрация свободных электронов в слоях (табл. 2). Прохождение концентрации электронов через максимум можно объяснить попаданием атомов иода с повышением его количества в другие, кроме $\mathrm{I}_{\text {Te }}$, положения в решетке полупроводника (комплексы, междоузлия, $\mathrm{I}_{\mathrm{Cd}}$ и др.), компенсирующие примесь или нейтральные. Степень компенсации иода также проходит через максимум (табл. 2).

Существенным фактором, влияющим на соотношение $\mathrm{Cd} / \mathrm{Te}$ на ростовой поверхности, является отношение ДМК/ДЭТ в газовой фазе. Осаждение $\mathrm{CdTe}$ в избытке ДМК приводит к уменьшению количества комплексов $\left[V_{\mathrm{Cd}}^{2-}-\mathrm{I}_{\mathrm{Te}}^{+}\right]^{-}$в слоях, уменьшению степени компенсации $\mathrm{I}_{\mathrm{Te}}^{+}$и увеличению концентрации свободных электронов (табл. 3 и рис. 5). Наличие максимума на зависимости $n$ от ДМК/ДЭТ при ДМК/ДЭТ $=2$ можно, к примеру, объяснить возрастанием взаимодействия в газовой фазе ДМК (при $V_{\mathrm{DMCd}}>4 \cdot 10^{-5}$ моль/мин) с ИПИ (при $V_{\mathrm{IPI}}>1 \cdot 10^{-6}$ моль/мин) с преждевременным осаждением $\mathrm{CdI}_{2}\left(T_{\text {subs }}=388^{\circ} \mathrm{C}\right)$ на стенки реактора выше пьедестала и уменьшением фактического соотношения $\mathrm{Cd} / \mathrm{Te}$ на ростовой поверхности. Уменьшение $\mathrm{Cd} / \mathrm{Te}$ приводит к падению вхождения иода в положение $\mathrm{I}_{\mathrm{Te}}^{+}$и уменьшению концентрации свободных электронов.

Температура зоны выше пьедестала $\left(T_{\text {prezon }}\right)$ влияет в интервале $290-320^{\circ} \mathrm{C}$ на термораспад ДМК и не влияет заметно на распад ДЭТ, что меняет фактическое соотношение $\mathrm{Cd} / \mathrm{Te}$ на ростовой поверхности, приводя к появлению максимума на зависимости $n$ от $T_{\text {prezon }}$ (рис. 6).

При температуре подложки $350^{\circ} \mathrm{C}$, видимо, в наших условиях осаждения создаются оптимальные соотношение $\mathrm{Cd} / \mathrm{Te}$ на ростовой поверхности и степень распада ИПИ, что объясняет образование максимума на зависимости концентрации свободных электронов от $T_{\text {grow }}$ (табл. 4).

При отжиге легированных слоев при $500^{\circ} \mathrm{C}$ в парах кадмия заполняются вакансии кадмия, разрушаются комплексы $\left[V_{\mathrm{Cd}}^{2-}-\mathrm{I}_{\mathrm{Te}}^{+}\right]^{-}$и освобождаются связанные доноры $\mathrm{I}_{\mathrm{Te}}^{+}$, приближая степень активации иода к 100\% (табл. 5). Сравнение с результатом отжига в тех же условиях нелегированного CdTe показывает, что концентрация электронов в легированном образце в первую очередь обусловлена ионизованным иодом, а не межузельным кадмием.

\section{4. Заключение}

Исследованы закономерности вхождения иода из изопропилиодида в MOVPE CdTe в зависимости от кристаллографической ориентации подложки, концентрации легирующего прекурсора, соотношения металлоорганических соединений, температуры роста и стенок реактора, а также влияние последующего термического отжига на концентрацию носителей заряда в слоях. Кристаллографическая ориентация слоев сильно влияет 
на вхождение и поведение иода. При увеличении потока легирующего прекурсора растет общее вхождение примеси в слои и концентрация электрически активного иода. Для каждой ориентации характерен свой ансамбль собственных дефектов и примесь иода, взаимодействуя с ним, по-разному распределяется между различными положениями в решетке. В работе в исследованных условиях осаждения обнаружены максимумы на зависимостях концентрации свободных электронов в слоях от потока изопропилиодида, соотношения ДМК/ДЭТ, температуры зоны прекрекинга и подложки. Достигнута концентрация некомпенсированных электронов в слоях CdTe (100) $1.1 \cdot 10^{16} \mathrm{~cm}^{-3}$ и $\mathrm{CdTe}(211) \mathrm{A} 3.6 \cdot 10^{16} \mathrm{~cm}^{-3}$. Степень активации иода составила от $\sim 0.02$ до $\sim 4 \%$. После отжига в парах кадмия при $500^{\circ} \mathrm{C}$ в течение 3 ч степень активации иода в слоях СdТе увеличилась до 100\%.

\section{Финансирование работы}

Работа выполнена по государственному заданию Министерства образования и науки РФ (тема № FFRN-2019-0004).

\section{Благодарности}

Авторы выражают благодарность М.Н. Дроздову за ВИМС исследования слоев, И.И. Евдокимову за проведение анализа слоев ICP АЕ методом.

\section{Конфликт интересов}

Авторы заявляют, что у них нет конфликта интересов.

\section{Список литературы}

[1] C. Szeles. Phys. Status Solidi B, 241 (3), 783 (2004).

[2] J.M. Burst, J.N. Duenow, D.S. Albin, E. Colegrove, M.O. Reese, J.A. Aguiar, C.-S. Jiang, M.K. Patel, M.M. AlJassim, D. Kuciauskas, S. Swain, T. Ablekim, K.G. Lynn, W.K. Metzger. Nature Energy, 1 (3), 1 (2016).

[3] В.С. Евстигнеев, А.В. Чилясов, А.Н. Моисеев, М.В. Костюнин. Неорг. матер., 55 (10), 1040 (2019).

[4] В.С. Евстигнеев, В.С. Варавин, А.В. Чилясов, В.Г. Ремесник, А.Н. Моисеев, Б.С. Степанов. ФТП, 52 (6), 554 (2018).

[5] R. Sporken, S. Sivananthan, K.K. Mahavadi, G. Monfroy, M. Boukerche, J.P. Faurie. Appl. Phys. Lett., 55 (18), 1879 (1989).

[6] C.D. Maxey, J.P. Camplin, I.T. Guilfoy, J. Gardner, R.A. Lockett, C.L. Jones, P. Capper, M. Houlton, N.T. Gordon. J. Electron. Mater., 32 (7), 656 (2003).

[7] А.В. Чилясов, А.Н. Моисеев, В.С. Евстигнеев, Б.С. Степанов, М.Н. Дроздов. Неорг. матер., 52 (12), 1284 (2016).

[8] V.S. Evstigneev, A.V. Chilyasov, A.N. Moiseev, M.V. Kostunin. TSF, 689, 137514 (2019).

[9] K. Yasuda, Y. Tomita, Y. Masuda, T. Ishiguro, Y. Kawauchi, H. Morishita, Y. Agata. J. Electron. Mater., 31 (7), 785 (2002).

[10] M. Niraula, K. Yasuda, R. Torii, Y. Higashira, R. Tamura, B.S. Chaudhari, T. Kobayashi, H. Goto, S. Fujii Y. Agata. J. Electron. Mater., 49 (11), 6996 (2020).
[11] P.Y. Su, R. Dahal, G.C. Wang, S. Zhang, T.M. Lu, I.B. Bhat. J. Electron. Mater., 44 (9), 3118 (2015).

[12] S. Murakami, T. Okamoto, K. Maruyama, H. Takigawa. Appl. Phys. Lett., 63 (7), 899 (1993).

[13] M. Niraula, T. Aoki, Y. Nakanishi, Y. Hatanaka. J. Cryst. Growth, 200 (1), 90 (1999).

[14] D. Brun-Le-Cunff, T. Baron, B. Daudin, S. Tatarenko, B. Blanchard. Appl. Phys. Lett., 67 (7), 965 (1995).

[15] N.C. Giles, J. Lee, D. Rajavel, C.J. Summers. J. Appl. Phys., 73 (9), 4541 (1993).

[16] R. Bhat, C. Caneau, C.E. Zah, M.A. Koza, W.A. Bonner, D.M. Hwang, S.A. Schwarz, S.G. Menocal, F.G. Favire. J. Cryst. Growth, 107, 772 (1991).

[17] M. Kondo, C. Anayama, T. Tanahashi, S. Yamazaki. J. Cryst. Growth, 124, 449 (1992).

[18] В.С. Евстигнеев, А.В. Чилясов, А.Н. Моисеев, С.В. Морозов, Д.И. Курицын. ФТП, 55 (1), 9 (2021).

[19] G.B. Stringfellow. J. Cryst. Growth, 75 (1), 91 (1986).

[20] D.V. Forbes, J.J. Coleman. MRS Online Proceedings Library (OPL), 340, 289 (1994).

[21] S. Seto, A. Tanaka, Y. Masa, M. Kawashima. J. Cryst. Growth, 117, 271 (1992).

[22] Y. Marfaing. TSF, 387, 123 (2001).

[23] J.E. Hails, D.J. Cole-Hamilton, W. Bell. J. Cryst. Growth, 145, 596 (1994).

[24] J.B. Mullin, S.J.C. Irvine, D.J. Ashen. J. Cryst. Growth, 55, 92 (1981).

Редактор Г.А. Оганесян

\section{Influence of growth conditions from metalorganic compounds on the preparation of $n$-CdTe epitaxial layers using isopropyl iodide}

\section{A.N. Moiseev, V.S. Evstigneev, A.V. Chilyasov, M.V. Kostunin \\ Devyatykh Institute of Chemistry of High-Purity Substances Russian Academy of Sciences, 603951 Nizhny Novgorod, Russia}

\begin{abstract}
The dependence of iodine incorporation in CdTe layers on the deposition conditions during metalorganic vapor phase epitaxy is investigated. The growth of the layers was carried out from dimethylcadmium and diethyltellurium in the hydrogen flow in a vertical reactor with a hot wall condition at a total pressure of $20 \mathrm{kPa}$. The total iodine concentration was determined by secondary ion mass spectrometry, the electrically active concentration was determined from the Hall effect measurement. The iodine incorporation depends on the crystallographic orientation of the substrate (were studied (100), (310), (111)A, (111)B, (211)A and (211)B), the concentration of the doping precursor (flux range $5 \cdot 10^{8}-3 \cdot 10^{6} \mathrm{~mol} / \mathrm{min}$ ), the mole ratio of organometallic compounds $(\mathrm{DMCd} / \mathrm{DETe}=0.25-4)$, growth temperature $\left(335-390^{\circ} \mathrm{C}\right)$ and the walls of the reactor above the pedestal (hot wall zone $290-320^{\circ} \mathrm{C}$ ). The total iodine concentration reached $5 \cdot 10^{18} \mathrm{~cm}^{-3}$ and the activation efficiency was $\sim 4 \%$. After thermal annealing in cadmium vapor at $500^{\circ} \mathrm{C}$ the activation efficiency was $\sim 100 \%$.
\end{abstract}

